\title{
Temperature Field Simulation of High Current Switchgear based on Fluent
}

\author{
Xiaojing Tian ${ }^{1, a}$, Huajun Dong ${ }^{1, b}$ and Liang Zhao ${ }^{1}$ \\ ${ }^{1}$ School of Mechanical Engineering, Dalian Jiaotong University, Dalian , 116028, China \\ atzy@djtu.edu.cn, bhuajundong4025@163.com
}

Keywords: High-current switchgear; Temperature Field Simulation; Fluent

\begin{abstract}
According to the overheating problem in the operation of high-current switchgear, numerical simulation of temperature field is analyzed based on Fluent. Mainly including the heat power calculation of heating elements in the switchgear, the heat transfer analysis and a three dimensional model of cooling system. The rated current is $4 \mathrm{KA}$ and the environment temperature is $40^{\circ} \mathrm{C}$, the thermal simulation analysis has been done respectively on the condition of totally enclosed,natural ventilation and forced ventilation schemes. It analyzes the relationship of maximum temperature in the vacuum switchgear with wind speed, different inlet position and heat dissipation modes, which can provide basis for the determination of the dissipation modes and the structure optimal design of high current switchgear.
\end{abstract}

\section{Introduction}

The overheat problem of high current switchgear can make the temperature of the elements too high which directly lead to the changes of physical and chemical properties of the material, decrease the mechanical and electrical performance, and even lead to the failure of the switchgear and may cause serious accidents[1].According to overheat problems,heat calculating and heat radiating systems designing have been becoming the very important researching fields of switchgear[2,3]. High current switchgears are the inevitable products in adapting to the trend of large capacity and high current power systems. It is very necessary to process the thermal designing and simulation analysis in guiding the high current switchgear designing .

Thermal simulation can perform the temperature field analysis under different heat transfer conditions from the aspects of convection, conduction and heat radiation.In recent years, many scholars have done a lot of research works in the field of temperature field simulation of switchgear. Zhang Junmin[4] of Tsinghua University established the mathematical model of 27.5kV GIS switchgear and simulated 3D temperature field based on the numerical calculation and analysis, which solved the coupling problem of temperature field and flow field in high voltage apparatus.

Zhou Yan[5] analyzed the temperature field of high pressure switch cabinet. Wang Bingzheng[6] analyzed the heating temperature of switch cabinet by ANSYS software .The isolating switch, bus and other parts of temperature rise were analyzed.

It is the basis of all to establish thermal models and to determine boundary conditions.Fluent is one of the most powerful means to analyze fluid problems and it is also an effective way in solving the above problems. The analysis of temperature field may find problems in products or in engineering designing. The application of Fluent simulation technology may shorten the designing cycle, reduce funds needs, provide the basis for the prediction of thermal stability and operating reliability of the switchgear.

This paper aims at the overheating problems of high current switchgear during operating situation. It analyzes the temperature and flow fields using the widely applicated software Fluent. Firstly, a three-dimensional model of high current switchgear is established using GAMBIT.Then is the simulation analysis of the temperature field through Fluent respectively under the conditions of full enclosed and ventilated. The main contents include the physical model establishment of the switch cabinet, meshing and boundary conditions setting, the material properties determination, 
calculations and the results' analysis. To investigate the structure designing and application for high current switchgears from the aspect of temperature and flow fields.

\section{Heat Transfer Models}

There are widespread flow and heat transfer problems in various fields. The direct power in generating heat flux is temperature difference. In nature, where there is temperature difference there is heat transfer from high to low temperature. The basic modes of heat transfer is nothing more than the three of heat conduction, convection and radiation [7].

The heat conduction of the switch cabinet mainly occurs in the current carrying conductors that contacts with the equipment or between the lap joints. And thermal radiation can be improved by improving the emissivity of the parts' surface. The heat conduction and radiation patterns are relatively stable in the switchgear. So what can lead the cooling effect of the switch cabinet is the thermal convection method.

In the flow and heat transfer problems, the variables, the convection and diffusion term in the governing equation can be processed to standard forms by mathematical processing, so as to simplify the general differential equation as the following form:

$$
\frac{\partial(\rho \phi)}{\partial t}+\operatorname{div}(\rho u \phi)=\operatorname{div}(\Gamma \operatorname{grad} \phi)+S
$$

\section{Heating Power Calculation}

Different kinds of heating losses constitute the internal heat sources in the actual operation of switchgears. The heating by dielectric loss is very small which can be ignored, while ferromagnetic heat loss can be effectively reduced through the measures of using non-magnetic material or non magnetic gap. Therefore, the resistance heating loss is considered mainly in temperature field analysis in this paper.

Buses, vacuum circuit breaker, isolating switch,earthing switch are the main heating elements in the cabinet.Table 1 shows the heating powers of components. The Joule loss of the buses mainly comes from the resistance heating loss of source currents. The bus resistance includes the resistance of current carrying conductors and the resistance of contacting. The power of bus radiating is calculated as the follow:

$$
P=I^{2} R=I^{2} \rho_{A L} \frac{L}{S_{A L}}
$$

in which

$$
\begin{aligned}
& \rho_{A L}-\text { copper bus resistance coefficient } \\
& L-\text { Bus length } \\
& S_{A L}-\text { Bus section } \\
& I-\text { Bus rated current }
\end{aligned}
$$


Table 1 Heating power of components

\begin{tabular}{|c|c|c|c|}
\hline Number & Components & $\begin{array}{c}\text { Heat Power } \\
(\mathrm{W})\end{array}$ & $\begin{array}{c}\text { Heat Flow Density } \\
(\mathrm{W} / \mathrm{m} 2)\end{array}$ \\
\hline 1 & bus & 282 & 433 \\
\hline 2 & vacuum circuit breaker & 879 & 359 \\
\hline 3 & current transformers & 9 & 27 \\
\hline 4 & grounding switch & 220 & 144 \\
\hline 5 & isolation switch & 230 & 251 \\
\hline
\end{tabular}

\section{Boundary Conditions and Meshing}

The dimensions of the switchgear body is $1500 \mathrm{~mm}$ length, $800 \mathrm{~mm}$ width, $1920 \mathrm{~mm}$ high. The high current bus is a bus bar combination of two plates of copper, the distance of which is $10 \mathrm{~mm}$. Other components are simplified according to their shape size. The model processes by a Boolean function so that the switch cabinet becomes a whole.

Meshes divided in GAMBIT, the first step is the surface triangle mesh division of each electrical components, then is the hexahedral mesh division. The general principle is to ensure the proper amounts, subdivision at the places where the changes of fluid field are great and coarse grid section where the changes are small[8]. The total grid number of this high current switchgear is 418850 . As shown in figure 1. Figure 2 shows the locally refined zoom grids of the buses where is subdivided.

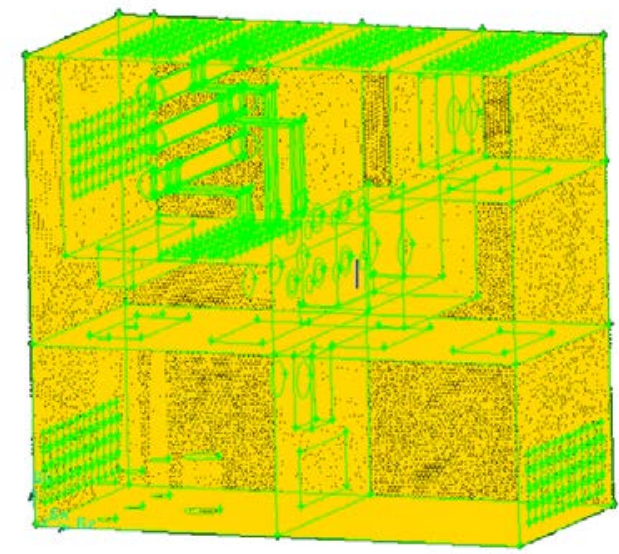

Fig.1 Meshes of the switchgear model

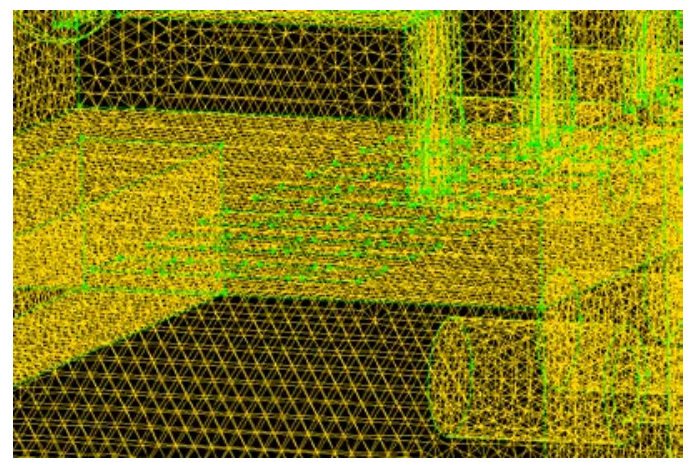

Fig.2 Locally refined zoom meshes

The inlet is set of speed inlet, and when the wind speed is 0 , the simulated temperature rise is the enclosed condition. The boundary type of the outlet is set of outflow, and the default setting is reserved. Bus, vacuum breaker, cable are all set of heating bodies, choosing heat flux density settings, and the values are set due to table 1.

Switchgear and cabinet provided with a no slip wall, made of stainless steel materials. At the same time, considering the convection and radiation heat transfer coefficient, air natural convection, radiation from the rate of the default value. The main heating element in the switch cabinet is taken for the wall condition, set the heating quantity of each element.This paper selects 
the standard $k-\varepsilon$ model to set the flow solids coupling in the switch cabinet, allowing the fluid and the wall to be coupled so that the heat can be transmitted.

\section{Temperature Field Simulation}

The simulation condition is that the current is $4 \mathrm{KA}$ and the environment temperature is $40^{\circ} \mathrm{C}$. The temperature rise between the double buses and the junction of bus and the contact exceeds the standard, as shown in figure 3. The maximum temperature rise of buses comes to 253K, the junction temperature rise of buses and the contact reaches $187 \mathrm{~K}$, which is seriously higher than the maximum allowable temperature rise $70 \mathrm{~K}$ of copper in air. As shown in figure 4 is the positions where the surface temperatures are seriously exceed the standard.

Therefore, under the condition of continuous high current, the enclosed high voltage switchgear will bring harm to the safe operation of the switchgear, and effective heat dissipation measures must be taken.

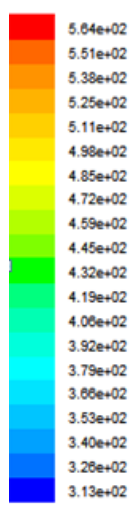

Fig.3 Temperatures of the connecting positions
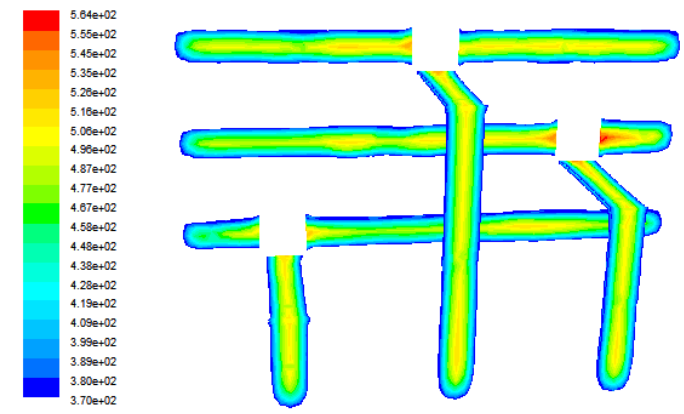

Fig. 4 Temperatures exceed standards

When the air flow speed is $925 \mathrm{~m} 3 / \mathrm{h}$ (the fan speed is $5.14 \mathrm{~m} / \mathrm{s}$ ), the ambient temperature is 40 degrees centigrade, as shown in Figure 5, the highest temperature rise is $64 \mathrm{~K}$. In double buses connection gap the maximum temperature rise is nearly within the allowed maximum temperature appreciation. Figure 6 shows the fluid field distribution in the cabinet, wind flows around the high vacuum circuit breaker, whereas the wind speed is lower around the buses.

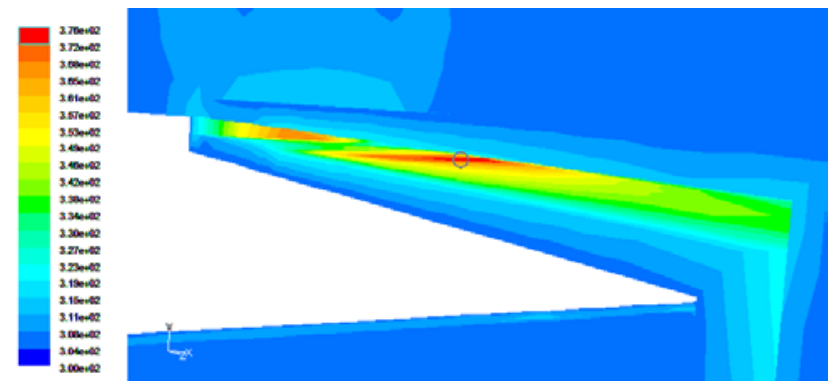

Fig.5 Amplification temperature map
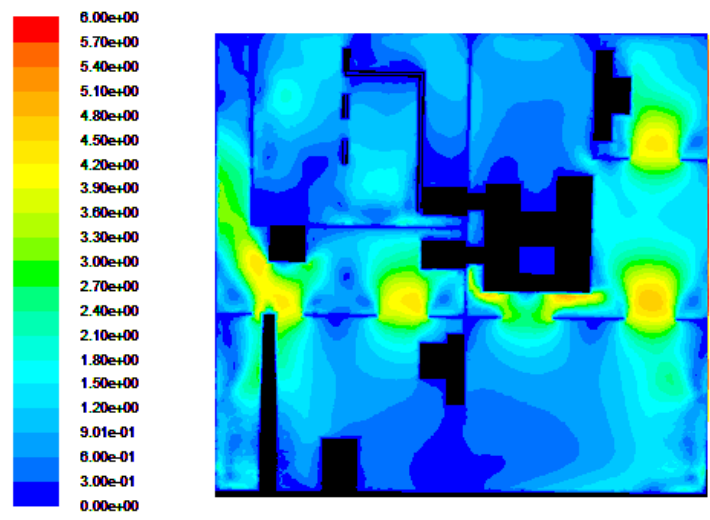

Fig. 6 Flow field distribution

Figure 7 shows the relationship of the maximum temperature(Tmax) and wind speed.Tmax drops with the increase of wind speed, ,but when the wind speed increases to a certain value, the temperature changes slowly. 
On the condition of natural ventilation,when the wind speed is lower than $3 \mathrm{~m} / \mathrm{s}$, the switch cabinet temperature rise decreased significantly, the local maximum temperature rise is $96 \mathrm{~K}$, which has reached the highest temperature rise requirement of copper bus in the air. When the wind speed is higher than $5.73 \mathrm{~m} / \mathrm{s}$, the maximum temperature has decreased, but the decline rate has slowed down. Therefore, through the method of simple increasing of ventilation capacity is not enough to solve the temperature rise problem in the switch cabinet.

Therefore, we have studied the relationship between the maximum temperature and wind speed at different inlet positions. As shown in Figure 8, the up air inlet scheme is better than that of down air inlet, the highest temperature in the switch cabinet has declined, especially when the wind speed is between $2 \mathrm{~m} / \mathrm{s}$ to $6 \mathrm{~m} / \mathrm{s}$. And the effect is obvious, the maximum temperature difference is about 20 degrees. But when the wind speed continues to increase, the two schemes do not show much difference.

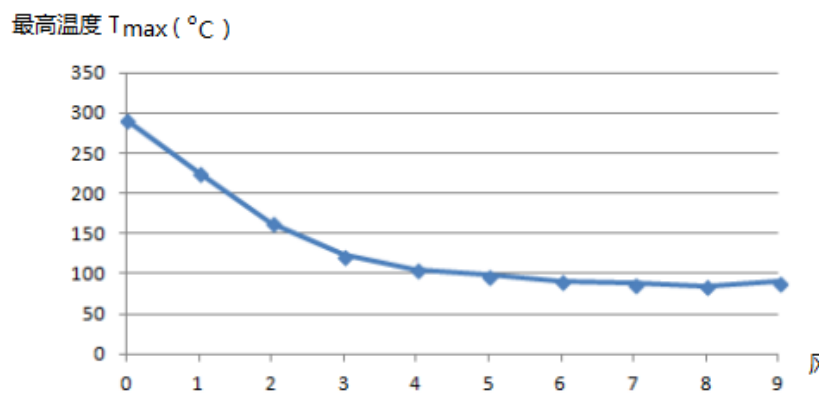

Fig.7 Tmax and wind speed relationship

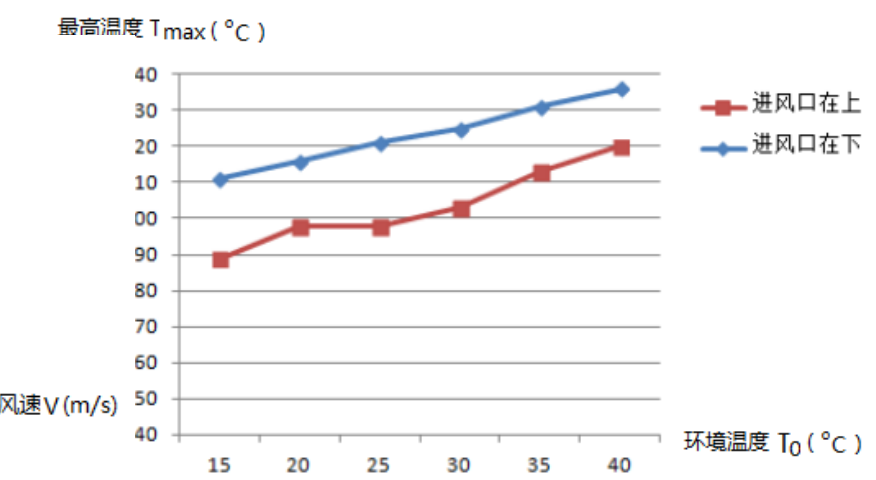

Fig. 8 Tmax of different inlet position

\section{Conclusion}

In this paper, aiming at the problem of heat dissipation of high current switchgear, it analyzes the temperature field of enclosed,natural ventilation and forced ventilation conditions through Fluent. The junction temperature of buses and circuit breaker seriously exceeds the standard at the enclosed condition.The air inlet position moves up near the bus room scheme is better than the air inlet at the bottom. When the wind speed increase to over $5.73 \mathrm{~m} / \mathrm{s}$, switch cabinet temperature can meet the requirements. The maximum temperature is at the inter-layer surface of the double buses, which shows the establishment of double bus structure model is more meaningful in the analysis of switch cabinet temperature field.

\section{Acknowledgement}

In this paper, the research was sponsored by National Natural Science Foundation of China (51477023).

\section{References}

[1] Huang hengxi. Application of the simple algorithm of high voltage switch cabinet and the technical measures to reduce the corresponding heat[J], Fujian architecture, 2008.

[2] Liu Changchun.Analysis and solving of heat dissipation of closed large current switch equipment[J].Hunan water conservancy and hydro-power, 2008.

[3]Chao Zhigang, Zhou Huidong, Yue Yanfeng. The heat and heat transfer of the flow circuit of high voltage switch device. [J]. Power and energy. 2009.

[4]Zhang Junmin,Hou Zhenhua, Zhang Chunpeng etc. The numerical calculation of the 3D 
temperature field of the $27.5 \mathrm{kV}$ GIS bus room [J]. Journal of electrical engineering, 2011, 12 (26) : 62-67

[5]Zhou Yan, Gao Yang, Liu Tingting, et al. Numerical simulation analysis of the temperature field of high pressure switch cabinet [J]. Sensors and Micro-systems, 2008,12 (7) : 102-105.

[6]Wang bingzheng, jiang jianwu, zhao ling, etc. High voltage switch cabinet contact heat temperature field numerical calculation [J]. High voltage appliance, 2010, 49 (12) : 42-48

[7]Tao Wenquan. Digital heat transfer [M]. Xi-an Jiaotong university press,1988.

[8] Wang Fujun. Computational fluid dynamics analysis [M]. Beijing: tsinghua university press, 2004. 